\title{
Coherence in complex networks of oscillators
}

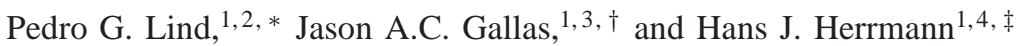 \\ ${ }^{1}$ Institute for Computational Physics, Universität Stuttgart, Pfaffenwaldring 27, D-70569 Stuttgart, Germany \\ ${ }^{2}$ Centro de Física Teórica e Computacional, Av. Prof. Gama Pinto 2, 1649-003 Lisbon, Portugal \\ ${ }^{3}$ Instituto de Física, Universidade Federal do Rio Grande do Sul, 91501-970 Porto Alegre, Brazil \\ ${ }^{4}$ Departamento de Física, Universidade Federal do Ceará, 60451-970 Fortaleza, Brazil
}

(Dated: November 21, 2018)

\begin{abstract}
We study fully synchronized (coherent) states in complex networks of chaotic oscillators, reviewing the analytical approach of determining the stability conditions for synchronizability and comparing them with numerical criteria. As an example, we present detailed results for networks of chaotic logistic maps having three different scale-free topologies: random scale-free topology, deterministic pseudo-fractal scale-free network and Apollonian network. For random scale-free topology we find that the lower boundary of the synchronizability region scales approximately as $k^{-\mu}$, where $k$ is the outgoing connectivity and $\mu$ depends on the local nonlinearity. For deterministic scale-free networks coherence is observed only when the coupling is heterogeneous, namely when it is proportional to some power of the neighbor connectivity. In all cases, stability conditions are determined from the eigenvalue spectrum of the Laplacian matrix and agree well with numerical results based on histograms of coherent states in parameter space. Additionally, we show that almost everywhere in the synchronizability region the basin of attraction of coherent states fills the entire phase space, and that the transition to coherence is of first-order.
\end{abstract}

\section{THE INTERPLAY BETWEEN DYNAMICS AND TOPOLOGY}

The structure and dynamics underlying complex networks have been widely investigated, providing insight for many systems where they arise naturally [1, 2, 3]. Complex networks appear in a wide variety of fields ranging from lasers [4], granular media [5, 6], quantum transport [7], colloidal suspensions [8], electrical circuits [9], and time series analysis [10], to heart rhythms [11], epidemics [12, 13], protein folding [14], and locomotion [15] among others [1, 2, 3].

From the mathematical point of view, a network is a graph, composed by nodes or vertices and by their connections or edges [2]. Sometimes, each node is characterized by some dynamical state (amplitude), which evolves according to some local contribution and to the interaction with the neighborhood. In other words, the complexity of the system underlying the network may be introduced either in the way nodes are interconnected (topology) or in the way nodes evolve in time (dynamics).

When studying network dynamics one frequently assumes a regular topology (lattice) where each node evolves according to some more or less complicated dynamics, typically fixed points [16], limit cycles [17] or chaotic attractors [18, 19]. One main goal of this approach is to study the so-called spatio-temporal chaos which appears in many different spatially extended systems out from equilibrium, such as hydrodynamical flows, chemical reactions and biological systems [20, 21]. Two main topics in this context concern the study of mechanisms underlying pattern formation and pattern selection [20, 21, 22, 23, 24] and also the study of chaotic

\footnotetext{
*URL:|http://www.ical.uni-stuttgart.de/ lind

†URL:http://www.if.ufrgs.br/〜jgallas

‡URL: http://www.ical.uni-stuttgart.de/ hans
}

synchronization behavior [16, 25].

Spatially extended systems are fundamentally modeled by (i) sets of coupled differential equations 20] with nonlinear terms, where both time and amplitude are continuous, (ii) cellular automata [22], where both time and amplitude are discrete or (iii) coupled map lattices[21], where time is discrete as in cellular automata, but the space of states is continuous. In all these models the underlying networks have connections whose range assumes all values from 1 (nearest neighbors) up to some maximum range, in particular the size of the system (global coupling regime). In other words, neglecting boundary conditions, these network systems assume translational symmetry and therefore the underlying network is called a regular network.

To study more complicated network structures, one usually neglects node dynamics and all complexity is introduced by the network topology, i.e. by the way nodes are connected to each other. This may be done in three different ways |2]: by randomly connecting the nodes (random networks [26, 27]), by considering some random long-range connections in a regular network with some small range of couplings (small-world networks [28, 29]), or by considering the introduction of new nodes which are connected to the previous ones following some rule of preferential attachment (scale-free networks [30]). For all these cases there is no translational symmetry and no typical range connection: connections do not have a 'spatial' interpretation. Therefore, one uses some general topological quantities to characterize each particular network, namely the average path length $\langle\ell\rangle$, i.e. the average minimum number of connections linking two nodes, the clustering coefficient $C$, defined as the average fraction of neighbors which are connected to each other, and the distribution of connections $P(k)$, representing the number of nodes having $k$ connections. Table $\llbracket$ shows the values of these three quantities for all three topologies.

Random networks were introduced by Erdös and Rényi in the late fifties [26] to study organizing principles underlying 


\begin{tabular}{cccc}
\hline & Random & Small-world & Scale-free \\
\hline$\langle\ell\rangle$ & $\ln N / \ln (p N)$ & $\begin{array}{c}N \text { for small } p \\
\ln N \text { for large } p\end{array}$ & $\ln N / \ln \ln N$ \\
$C$ & $\bar{k} / N$ & $C_{0}(1-p)^{3}$ & $\sim N^{-3 / 4}$ \\
$P(k)$ & $\mathrm{e}^{-\bar{k}} \bar{k}^{k} / k !$ & $\mathrm{e}^{-\bar{k}} \bar{k}^{k} / k !$ & $2 m^{2} / k^{3}$ \\
\hline
\end{tabular}

TABLE I: Characterizing complex topologies with the topological quantities: average path length $\langle\ell\rangle$, clustering coefficient $C$ and distributions $P(k)$ of connections $k$. Here $N$ is the total number of nodes, $p$ is the probability for two nodes to be connected, $\bar{k}$ is the average number of connections per node, $C_{0}$ is the clustering coefficient of the regular network from which the small-world network is constructed, and $m$ is the number of initial connections of each new node in a scale-free network.

some real networks [27]. In random networks one defines some probability $p(N)$, function of the total number $N$ of nodes, which determines the probability for any two nodes to be connected in a total of $N$ nodes. Consequently, the connections are typically long-range connections having a completely irregular structure. One main goal in studying random networks is to determine the critical probability $p_{c}(N)$, beyond which some specific property starts to be very likely to be observed, e.g. the critical probability marking a transition to percolation [31]. One important feature of random networks, which also appears in real networks, is their small average path length $\langle\ell\rangle$, i.e. the average distance between any two nodes increases slowly with the system size. However, unlike random networks, real networks also have large cluster coefficients $C$.

Small-world networks were introduced recently by Watts and Strogatz [28] in order to satisfy both these two features, short $\langle\ell\rangle$ and large $C$. Small-world networks have short-range connections between neighbors, as in regular networks, but they also have long-range connections similar to random networks, without middle range ones. There are mainly two procedures to construct a small-world network: starting from same regular network, where each site is coupled to some number of first neighbors, one either rewires each regular connection with probability $p$ (Watts-Strogatz model [28]) or adds a random connection to each node with probability $p$ (Newman-Watts model[32]). The second procedure is more appropriate for most purposes, since it avoids the possibility of generating disconnected clusters 32].

Both random and small-world topologies produce typically networks where connections obey a Poisson distribution (see Tab. \. However, there are real systems which are scale-free, i.e. where the connection distribution obeys a power-law.

Scale-free networks were introduced by Barabási and Albert [30] using growing and preferential attachment: one starts with a small amount of nodes totally interconnected, and adds iteratively one node with $m$ connections to the previous nodes, chosen from a probability function proportional to their number of connections. With this construction one obtains analytically[33, 34, 35, 36] a distribution of the connections $P(k) \propto k^{-\gamma}$, where $\gamma \rightarrow 3$ as $N \rightarrow \infty$, independently of the initial number of fully interconnected nodes and of $m$.
It is also possible to generate scale-free networks, by either imposing a priori a power-law distribution of all connections randomly distributed, or by following a deterministic iterative rule for new nodes. The first procedure generates what is usually called a generalized random graph, while the latter was recently referred as deterministic scale-free network [37].

Deterministic scale-free networks, are hierarchical structures composed by some succession of generations of nodes, i.e. the set of new nodes appearing simultaneously at a given iteration during the 'construction' of the network, whose connections follow a particular power-law distribution [37, 38, 39, 40], being more easier to handle. The main difference between random and deterministic scale-free networks is due to the local connectivity character of the latter: random constructions generate irregular long-range connections, while deterministic networks impose a succession of generation of new nodes which are, in some way, organized in 'space'. Therefore, deterministic networks are applied for instance in spin systems [39], and geographical and social networks [39, 41].

After considering separately dynamical and topological complexity, the next logical step toward real network dynamics is to consider them together. One important question addressed in this context is to know if full synchronization between oscillators in such complex topologies would appear and under which conditions it is stable. By full synchronization we mean the convergence of the amplitudes of all oscillators to the same value, evolving coherently from then on. Therefore we call henceforth these fully synchronized states coherent states, to distinguish them from partially synchronized configurations, when several different clusters of nodes with the same amplitude are observed [16]. Synchronization and coherent behavior of oscillator networks with complex topologies have been studied for the random topology [42, 43, 44, 45] and small-world topology [46, 47, 48, 49], and also scale-free networks [45, 50, 51, 52, 53]. In random networks, it is already known [42] that with high coupling strengths it is possible to fully synchronize oscillators and the corresponding stability condition may be computed [43] from the matrix of connections characterizing the network. In small-world networks, synchronizability is observed [47] only at the end of the small-world regime (high values of $p$ ), and recently [46] it was found that heterogeneity in the coupling may destroy coherence. These findings for small-world networks are somehow controversial with the ones of other studies [45, 48] and other topological quantifiers have been proposed [49]. In scale-free networks some recent studies indicate that synchronizability among oscillators depends on the average connectivity [52] and is robust to delayed flow of information [50] and to the removal of low-connected nodes [52].

In this manuscript we describe the general approach to study coherent states in any general complex network of oscillators, and apply it to the particular case of a scale-free network of discrete-time oscillators, which is studied in great detail. We start in Section $\amalg$ by describing the stability analysis approach to model in Eq. (1) and deduce the corresponding conditions for synchronizability. In Section $\coprod$ we apply this stability analysis procedure to the particular case of scale-free 
networks, comparing our results with numerical simulations. The random scale-free case is treated in Section IIIA where we show that the threshold value of such a transition as a function of coupling strength and outgoing connectivity obeys a power-law with an exponent that depends on the nonlinearity, while deterministic scale-free networks are studied in II B namely a pseudo-fractal network [38] and an Apollonian network [39, 40]. Discussion and conclusions are given in Section IV

\section{GENERAL APPROACH TO ANALYZE COHERENT STATES}

For all the network topologies described above, if one considers discrete-time oscillators, namely maps of the interval, the equation of evolution for their amplitudes reads

$$
\vec{x}_{t+1}=\vec{f}\left(\vec{x}_{t}\right)-\varepsilon \mathbb{G} \vec{g}\left(\vec{x}_{t}\right),
$$

where $\varepsilon$ is the coupling parameter, $t$ labels time, $\vec{x}_{t}=$ $\left(x_{t, 1}, \ldots, x_{t, N}\right)$ with $x_{t, i}$ representing the amplitude at timestep $t$ of node $i=1, \ldots, N$, where $N$ is the total number of nodes, $\vec{f}=\left(f\left(x_{1}\right), \ldots, f\left(x_{N}\right)\right)$ and $\vec{g}=\left(g\left(x_{1}\right), \ldots, g\left(x_{N}\right)\right)$ with $f$ and $g$ being real nonlinear functions, and $\mathbb{G}$ is the coupling (Laplacian) matrix, whose element $G_{i j}$ represents the relative strength with which node $i$ is coupled to node $j$, and satisfies the conditions $\sum_{j=1}^{N} G_{i j}=0$ and $G_{i i}=1$ for all $i=1, \ldots, N$. In general $\mathbb{G}$ is a non symmetric matrix.

Usually, one choses $\vec{g}(\vec{x}) \equiv \vec{x}$ when studying linear coupling, and $\vec{g}(\vec{x}) \equiv \vec{f}(\vec{x})$ when studying nonlinear coupling. Here we consider the nonlinear case. Apart from this choice, all the information about dynamics is introduced in function $\vec{f}(\vec{x})$, while all the information about the coupling topology (regular, random, small-world or scale-free) and the coupling regime (either homogeneous or heterogeneous) is included in the coupling matrix $\mathbb{G}$.

From Eq. 11 one easily sees that the coherent state $x_{t, 1}=$ $x_{t, 2}=\cdots=x_{t, N}=X_{t}$ evolves in time according to the local map $X_{t+1}=f\left(X_{t}\right)$. There are two ways to study these coherent states: either by studying the stability of small perturbations of the coherent states or by making statistics over significant large samples of initial configurations, counting how many converge to a coherent state. Some attention to the parameter ranges must be taken, since the basin of attraction of the coherent states may be bounded by regions of phase space where amplitudes diverge. In particular, for maps of the interval one has $0 \leq \varepsilon \leq 1$ in order to guarantee convergence of any initial configuration.

In this manuscript we will illustrate both analytical and numerical approaches for the particular case of scale-free networks. To this end, we define the coupling matrix as $G_{i i}=1$ and

$$
G_{i j}=-\frac{k_{j}^{\alpha}}{\sum_{k \in \mathcal{K}_{i}} k_{k}^{\alpha}}
$$

if node $i$ is coupled to node $j$, with $k_{j}$ representing the number of nodes of node $j$ and $\mathcal{K}_{i}$ is the set of labels of all neighbors of node $i$. If nodes $i$ and $j$ are not coupled $G_{i j}=0$. The parameter $\alpha$ is a real number controlling the heterogeneity in the coupling: positive values of $\alpha$ enhance the coupling strength with sites having larger number of neighbors, while negative values favor sites having less neighbors. For $\alpha=0$ the coupling between each site and its neighborhood is homogeneous.

For local dynamics we choose the well-known quadratic map $f(x)=1-a x^{2}$, where the free parameter $a$ is restricted to the interval $-0.25 \leq a \leq 2$ and contains all possible dynamical regimes from a fixed point (e.g. $a=0$ ) to fully developed chaotic orbits (e.g. $a=2$ ).

When determining the stability of coherent states, various criteria are possible. For instance, one could compute the maximum Lyapunov exponent and obtain the conditions where it is negative. However, maximum Lyapunov exponents do not indicate the existence of local instabilities in the synchronous state, which may pull the trajectories apart from the coherent manifold.

The correct approach is based on the variational equation of Eq. (11) proposed by Pecora and Carroll [54], which is valid for any network of identical oscillators in what concerns their local dynamics (quadratic map, Lorenz system, etc.) and their coupling regime (linear, nonlinear, etc). For the nonlinear coupling regime, the diagonal form of these variational equations reads [54, 55, 56]

$$
\xi_{t+1, i}=\exp \left(\Lambda\left(\varepsilon \lambda_{i}\right)\right) \xi_{t, i}=\left[D f(X)-\varepsilon \lambda_{i} D f(X)\right] \xi_{t, i},
$$

for coherent states $x_{t, i}=X$, where $\Lambda\left(\varepsilon \lambda_{i}\right)$ is the Lyapunov exponent, $D f(X)$ represents the identity matrix multiplied by the derivative of $f(x)$ computed at $x=X$ and $\lambda_{i}$ are eigenvalues of the coupling matrix $\mathbb{G}$. If $\mathbb{G}$ has zero-sum rows, i.e. $\sum_{j=1}^{N} G_{i j}=0 \forall i$, and all its eigenvalues $\lambda_{1} \leq \lambda_{2} \leq$ $\cdots \leq \lambda_{N}$ are real and nonnegative, then $\lambda_{1}=0$ corresponds to the mode parallel to the synchronization manifold and the largest Lyapunov exponent defines a master stability function [54]. The coherent state is stable whenever $\Lambda\left(\varepsilon \lambda_{i}\right)<0$ for $i=2, \ldots, N$ [54, 55, 56].

In our case, it is easy to check from Eq. 21 that indeed $\mathbb{G}$ have zero-row sum, yielding $\lambda_{1}=0$. Moreover, all the eigenvalues of matrix $\mathbb{G}$ are real and non-negative, since $\operatorname{det}(\mathbb{G}-\lambda \mathbb{I})=\operatorname{det}(\overline{\mathbb{G}}-\lambda I \mathbb{I})$ where $\overline{\mathbb{G}}$ is a positive semidefinite symmetric matrix, namely $\bar{G}=\mathbb{H}^{1 / 2} \mathbb{K}^{1 / 2} \mathbb{A} \mathbb{K}^{1 / 2} \mathbb{H}^{1 / 2}$ with $\mathbb{A}$ being the adjacency matrix of the network [52], and matrices $\mathbb{H}$ and $\mathbb{K}$ being the diagonal matrices with elements $H_{i i}=1 /\left(\sum_{k \in \mathcal{K}_{i}} k_{k}^{\alpha}\right)$ and $K_{i i}=k_{i}^{\alpha}$ respectively.

From Eq. 3) and regarding the ordering of the eigenvalues $\lambda_{i}$ one easily concludes that the stability condition reads

$$
\varepsilon_{L} \equiv \frac{1-\exp (-\bar{\lambda})}{\lambda_{2}}<\varepsilon<\frac{1+\exp (-\bar{\lambda})}{\lambda_{N}} \equiv \varepsilon_{U},
$$

where $\bar{\lambda}$ is the Lyapunov exponent of the local single map. In particular there is a range of coupling strengths enabling synchronizability whenever $\lambda_{N} / \lambda_{2}<\left(1+\mathrm{e}^{-\bar{\lambda}}\right) /\left(1-\mathrm{e}^{-\bar{\lambda}}\right)$ holds. Therefore, by computing the eigenvalues of the Laplacian matrix $\mathbb{G}$ one is able to find the range of couplings for which coherent states are stable. This approach can be applied for any system ruled by Eq. (1). 

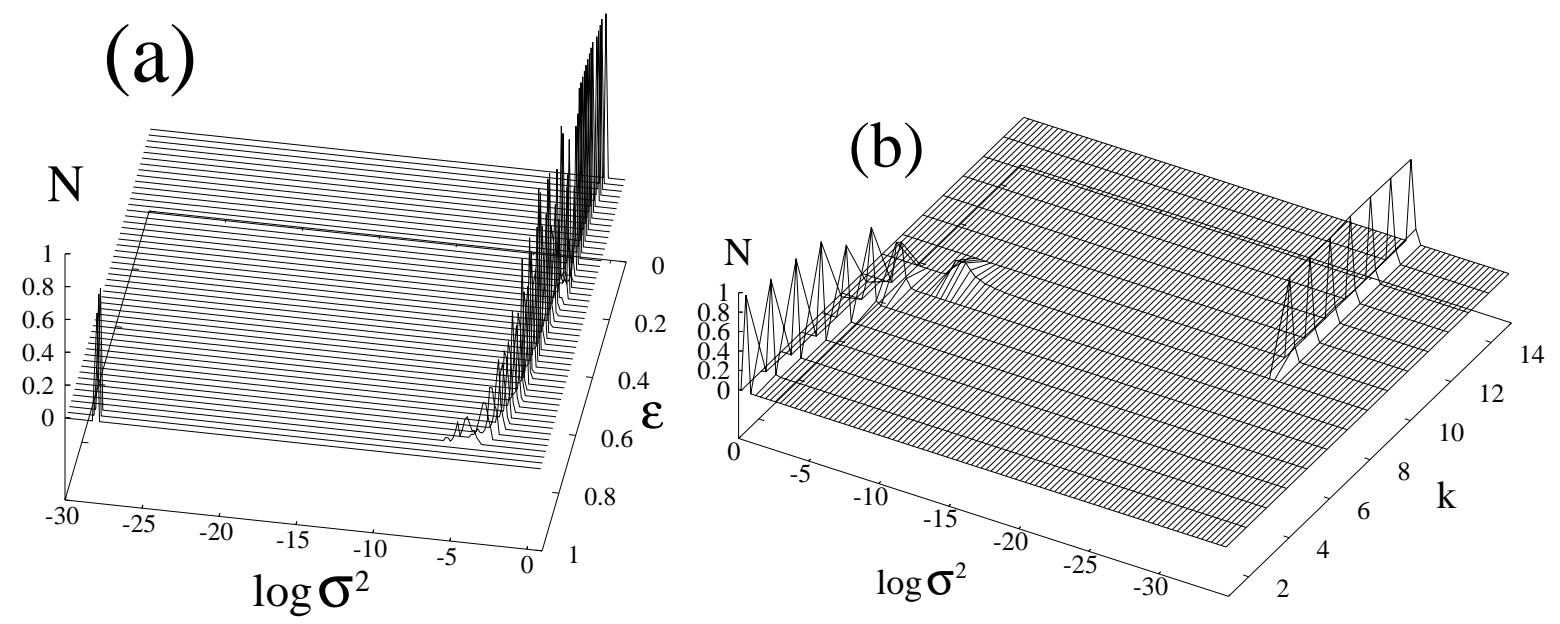

FIG. 1: Typical histograms of the standard mean square amplitude deviation $\sigma^{2}$ showing the sharp transition to coherence as a function of (a) the coupling strength $\varepsilon$ with $k=m_{0}=8$, and (b) the outgoing connectivity $k$ with $\varepsilon=0.95$. Values of $N$ represent the fraction of configurations which converge to a coherent state from a total of 500 initial random configurations, after discarding transients of $10^{4}$ time steps. Here $a=2, N=1000$, and $\alpha=0$.

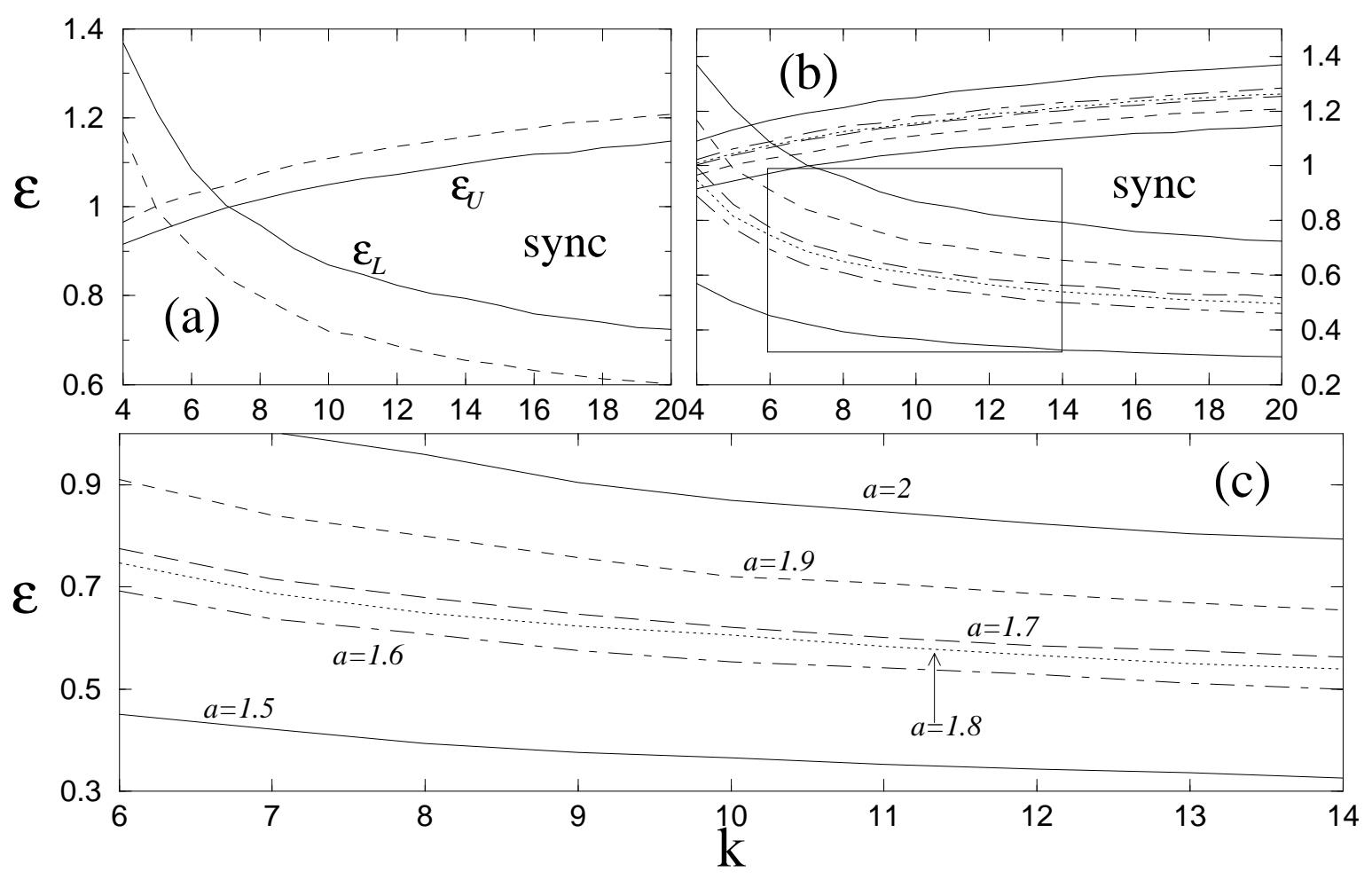

FIG. 2: Boundary values $\varepsilon_{L}$ and $\varepsilon_{U}$ in Eq. 4 as a function of connectivity $k$ (a) for $a=1.9$ (dashed lines) and $a=2$ (solid lines), and (b) for $a=1.5,1.6,1.7,1.8,1.9$ and 2, whose inset is magnified in (c). The regions labeled with 'sync' are the ones where coherent solutions are observed, i.e. $\varepsilon_{L}<\varepsilon_{U}$. Notice that in (c) the boundary for $a=1.8$ is below the one for $a=1.7$, contrary to other values (see text).

\section{SCALE-FREE NETWORKS OF COUPLED LOGISTIC MAPS: AN EXAMPLE}

For the particular case of scale-free networks, recent results [45] show a transition to full synchronization for two partic- ular values of the nonlinearity $a$ in the homogeneous regime ( $\alpha=0$ ), when either the coupling strength or the number of outgoing connections are varied. However, as far as we know there is no detailed study showing how these coherent states depend on all the parameter models. Therefore, we present 
in this Section detailed numerical results concerning synchronization in oscillator networks with scale-free topologies. Our purpose is to give a complete example of how to study coherent solutions in complex networks of oscillators, comparing both the stability analysis and the numerical approaches.

The stability analysis is carried out just by computing the boundary values $\varepsilon_{L}$ and $\varepsilon_{U}$ in Eq. (4) as a function of the model parameters. The ranges of values where $\varepsilon_{L}<\varepsilon_{U}$ are the ones where coherent solutions appear. As stated above, for discrete oscillators, ruled by maps of the interval, the condition $0 \leq \varepsilon \leq 1$ must be added.

Numerically, to detect coherent solutions from a given sample of initial configurations, we compute the standard mean square deviation [45]

$$
\sigma_{t}^{2}=\frac{1}{N} \sum_{i=1}^{N}\left(x_{t, i}-\bar{x}_{t}\right)^{2}
$$

where $\bar{x}_{t}$ is the average amplitude at a given time step $t$. Whenever $\sigma^{2}$ is zero within numerical precision, i.e. $\sigma^{2} \sim$ $10^{-30}$, all the nodes are synchronized at the same amplitude.

We divide our approach in two parts, the first one concerning random scale-free networks (Section $\amalg$ A) and a second one concerning deterministic scale-free networks (Section

\section{A. Random scale-free networks}

In this Section, we use the algorithm of Barabási and Albert [2, 30] to construct the random scale-free network (see Section 【, where at each node one places a chaotic logistic map. In a previous work [45] a transition to coherence between chaotic logistic maps was found for random scale-free networks, occurring for particularly high coupling strengths, typically of the order of $\varepsilon_{c} \sim 0.9$. Our simulations have shown that these transitions occur after discarding transients of $\sim 10^{4}$ time steps and they do not change significantly with the network size. Moreover, as shown in Fig. 10 this transition to coherence is robust with respect to initial configurations, either by varying the coupling strength $\varepsilon$ (Fig. 1. 1 ) or the outgoing connectivity $k$ (Fig. 1 b). In particular, above the threshold $\varepsilon_{c} \sim 0.9$, all initial configurations converge to a coherent state, indicating that in this parameter region the basin of attraction of coherent states fills almost the entire phase space.

From stability analysis, we find that in the fully chaotic regime $(a=2)$ the transition to coherence occurs for gradually smaller coupling strength if the connectivity $k$ is increased. Figure $2 \mathrm{~h}$ shows the boundaries $\varepsilon_{L}$ and $\varepsilon_{U}$ as a function of $k$ for $a=2$ (solid lines) and $a=1.9$ (dashed lines) with the same parameter values as in Fig. 11 As one sees, in both cases the lower boundary $\varepsilon_{L}$ decreases when $k$ increases, while the upper boundary $\varepsilon_{U}$ increases beyond $\varepsilon=1$. Therefore, one expects that the region of synchronizability increases for larger values of connectivity $k$. Figure 2 also shows clearly that for $a=2$ the intersection between both boundaries, $\varepsilon_{L}=\varepsilon_{U}$, occurs just above $k=7$, which explains why the transition to coherence in Fig. 1b occurs at this value. For $a=1.9$ this transition should occur near $k=5$. Decreasing even more the nonlinearity coherent solutions are observed for even smaller connectivities and synchronizability regions increase, as shown in Fig. 2 $\mathrm{b}$. To see this feature more clearly we magnify in Fig. 22 the inset of Fig. 2 2 . As one sees, one exception occurs for $a=1.8$, where the lower boundary is below the one for $a=1.7$, due to the fact that for
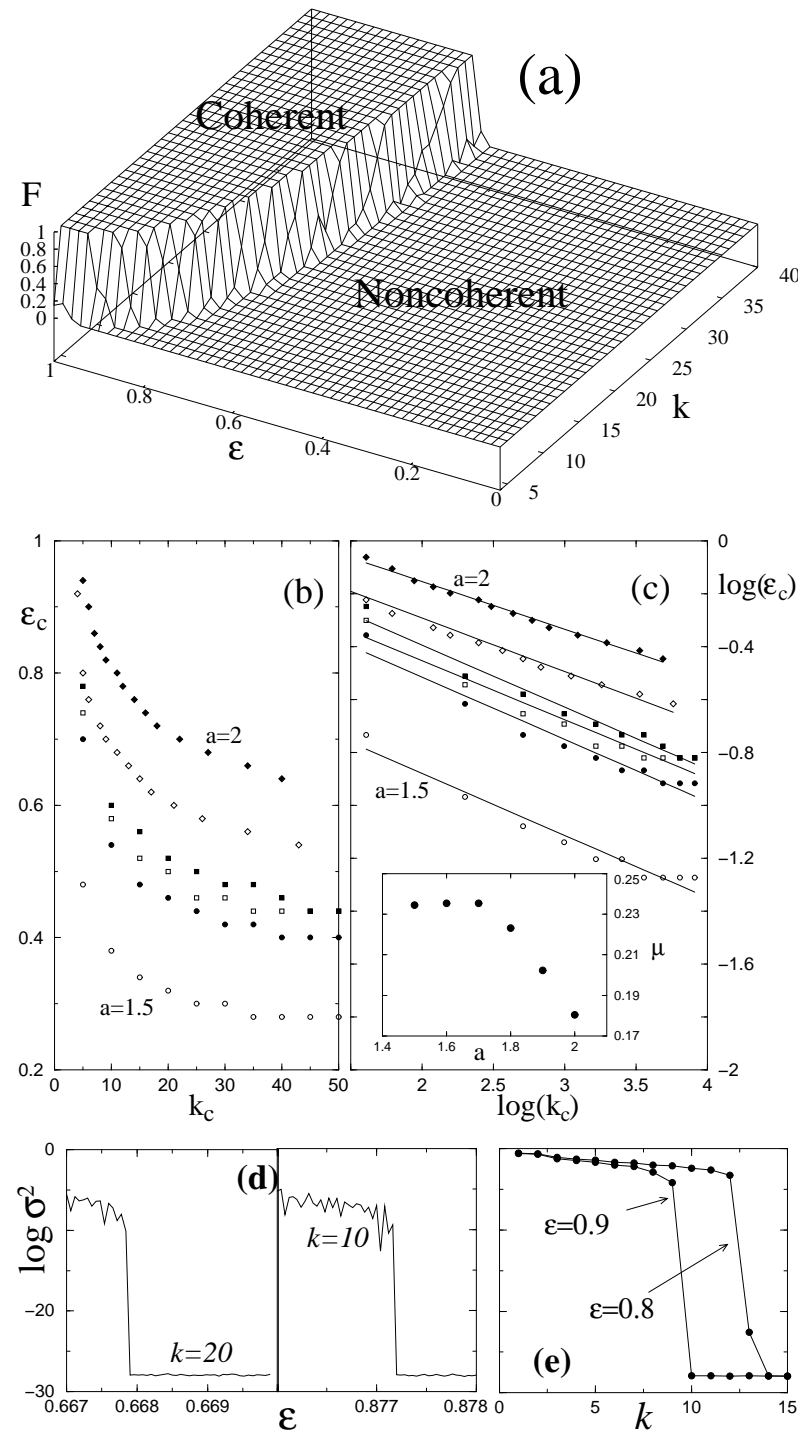

FIG. 3: Transition to coherence as a function of connectivity $k$ and coupling strength $\varepsilon$. (a) Fraction $N_{\sigma=0}$ of coherent states from 500 random initial configurations for $a=2$. (b) Coherence transition curves in the $(\varepsilon, k)$ plane for (from bottom to top) $a=1.5,1.6,1.8,1.7,1.9$ and $a=2$, and (c) the same transition in a log-log plot, showing power-law dependence between connectivity and coupling strength for the transition curves, with an exponent $\mu$ which depends on the value of $a$ (see inset). Here $\alpha=0, L=1000$ and we used transients of $10^{4}$ time steps. By increasing the transient size to $\sim 10^{6}$ one sees clearly that the transition to coherence is of first-order either (d) when varying the coupling strength $\varepsilon$ or (e) when varying the outgoing connectivity $k$. 


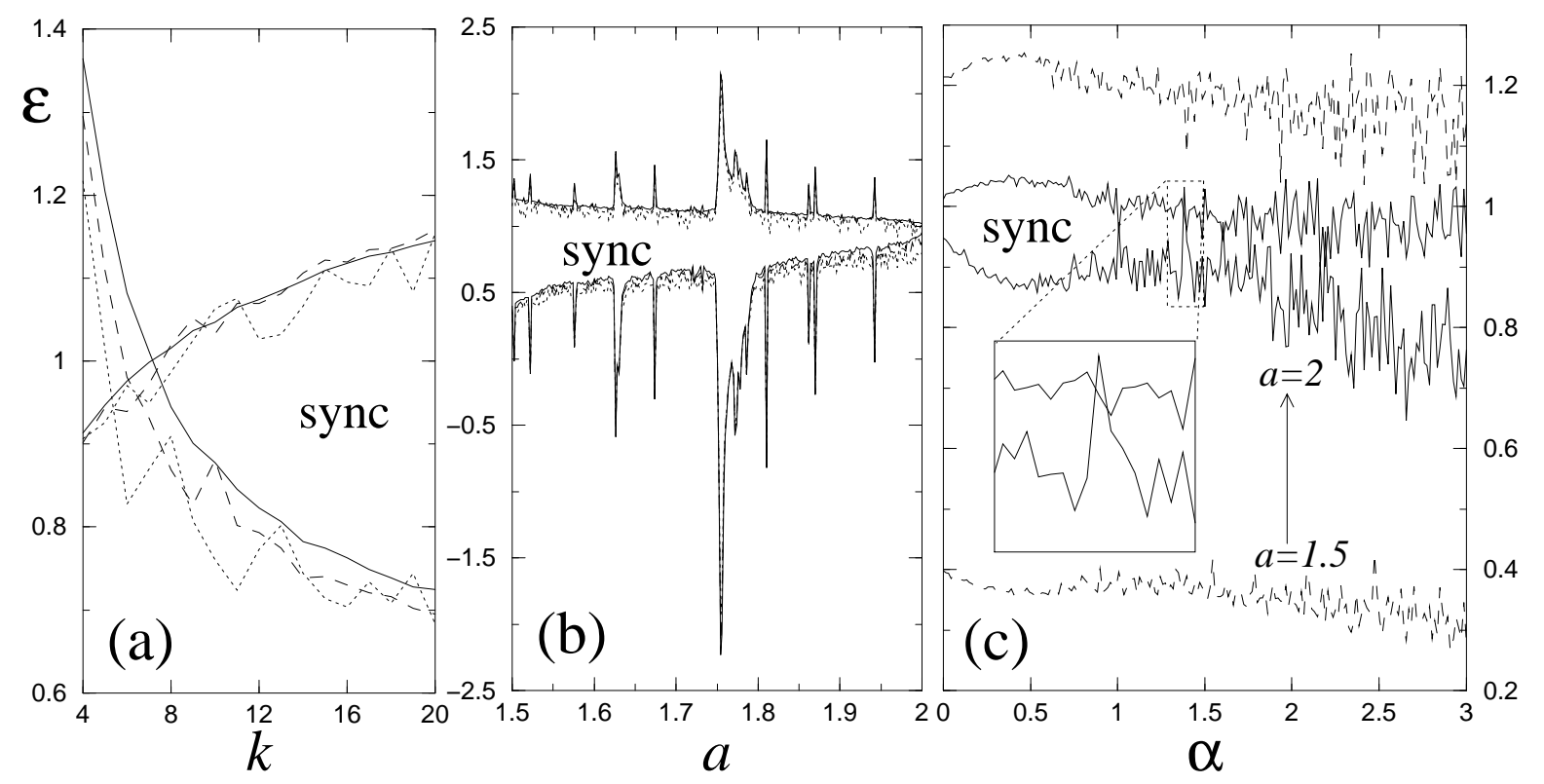

FIG. 4: Boundary values $\varepsilon_{L}$ and $\varepsilon_{U}$ in Eq. 4 as a function of (a) connectivity $k$ with $a=2$ and $\alpha=0$ (solid lines) $\alpha=1$ (dashed lines) and $\alpha=2$ (dotted lines), (b) nonlinearity $a$ for $k=8$ and $\alpha=0,1$ and 2, and (c) heterogeneity $\alpha$ with $k=8$ and $a=2$ (solid lines) and $a=1.5$ (dashed lines). The inset in (c) emphasizes one small region where synchronizability is not observed, $\varepsilon_{L}>\varepsilon_{U}$ (see text). Here $N=1000$.

$a=1.8$ the Lyapunov exponent of the logistic map is smaller

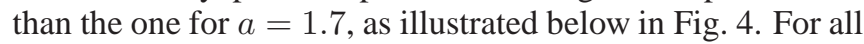
these values of $a$, the single uncoupled map shows chaotic orbits. Moreover, for any other network size $N$, the same curves are obtained.

These analytical predictions extracted from the stability condition in Eq. (4) and shown in Fig. 2 are strongly corroborated with our numerical simulations as shown in Fig. 3 In Fig. 33 we plot the fraction $F$ of initial configurations which converge to a coherent state for $a=2$, while Fig. 3b shows the threshold values, $\varepsilon_{c}$ and $k_{c}$, at the transition curves where the entire sample of initial configurations converge to a coherent state, for the same values of $a$ as in Fig. 22 r. Here, one clearly sees that there is a clear and sharp transition to coherence. Interestingly, the curves in Fig. 3. B fit very well the ones in Fig. 2.25, which means that whenever the synchronizability condition $\varepsilon_{L}<\varepsilon_{U}$ holds, coherent states fill almost entirely the phase space.

Moreover, as illustrated in Fig. 3r, all curves obey a powerlaw, within our numerical precision,

$$
\varepsilon_{c} \propto k_{c}^{-\mu} .
$$

For the six above values of $a$, the exponents are respectively $\mu=0.2345,0.2354,0.2353,0.2231,0.2023$ and 0.1804 : the exponent is almost constant below $a \sim 1.7$ and decreases above this value, as illustrated in the inset of Fig. 3.

To determine the nature of the transition to coherence seen in Figs. 3 and 3b, we plot in Figs. 3 and 3r the average standard deviation in the region where transition to coherence is observed, using much higher resolution. One clearly sees that the transition to coherence is of first-order, either when varying $\varepsilon$ or $k$. That the transitions are indeed of first order is easily recognized by the clear existence of hysteresis: when increasing either $\varepsilon$ or $k$ the configuration eventually falls into a coherent state, no longer spontaneously desynchronizing, no matter how far the parameters are tuned back.

All results till now, concern the case of homogeneous coupling $(\alpha=0)$. Next, we study the case of heterogeneous coupling. Figure 4 shows the boundaries $\varepsilon_{L}$ and $\varepsilon_{U}$ in Eq. (4) as a function of outgoing connectivity $k$, nonlinearity $a$ and heterogeneity $\alpha$, covering both the homogeneous and heterogeneous regimes. Figure $4 \mathrm{~h}$ shows the two boundaries as a function of $k$ for $a=2$ and $\alpha=0$ (solid lines), $\alpha=1$ (dashed lines) and $\alpha=2$ (dotted lines). As one sees for nonzero values of $\alpha$ the boundaries are no longer smooth curves, but instead they show fluctuations as $k$ is increased, enlarging and shrinking alternately the region of synchronizability, labeled as 'sync'. When varying $a$ (Fig. 4b) the boundaries are mainly controlled by the Lyapunov exponent of the local map (see Eq. (4)), where $\varepsilon_{L}$ (resp. $\varepsilon_{U}$ ) decreases (resp. increases) whenever a periodic window occurs [57]. The fluctuations observed in Fig. 固 are clearly seen in Fig. 4k, where the stability boundaries are plotted as a function of $\alpha$ fixing $k=8$ and $a=2$ (solid lines) and $a=1.5$ (dashed lines). The fluctuations are much higher for $\alpha>1$ and for the fully chaotic regime both boundaries may even cross each other suppress-

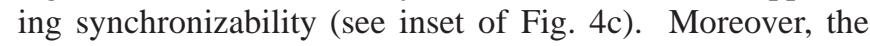
lower boundary $\varepsilon_{L}$ decreases till $\alpha \sim 0.5$, then increases till $\alpha \sim 1$ and decreases in average from there on.

All these analytical results computed from Eq. (4) and matrix $\mathbb{G}$ in Eq. $\mathbb{1 1}$ are corroborated by our numerical simulations. In particular, the boundaries $\varepsilon_{L}$ and $\varepsilon_{U}$ seen in Fig. 4 4 are obtained also when plotting the contour of Fig. 5h, where we plot the average standard deviation from a sample of 500 

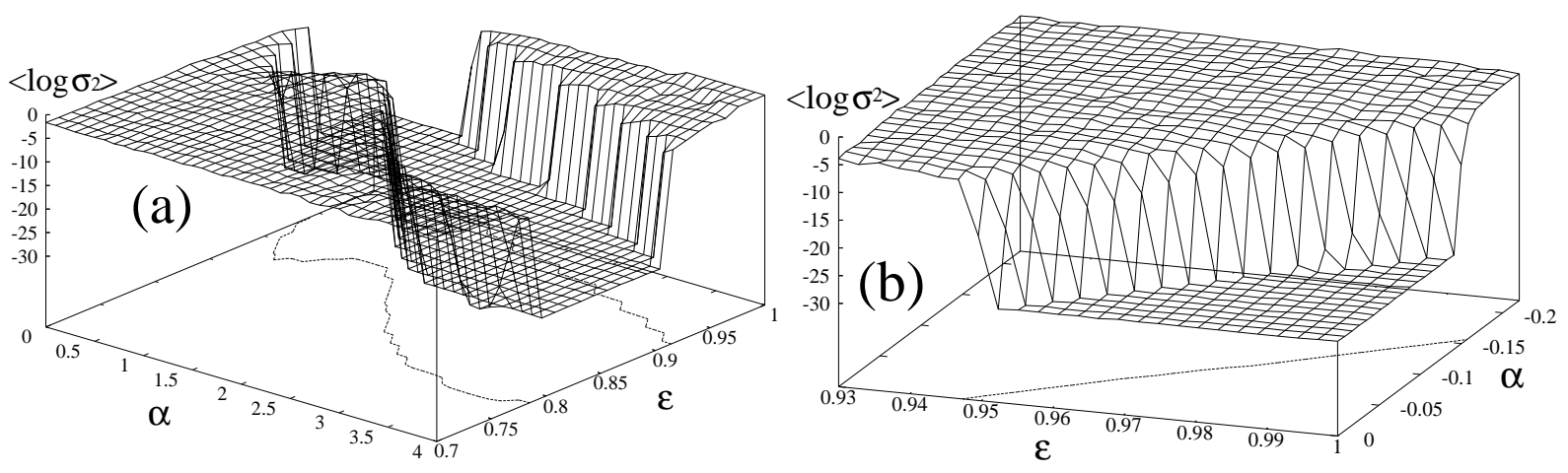

FIG. 5: Transition to coherence as a function of the coupling strength $\varepsilon$ and heterogeneity $\alpha$ (a) when most connected nodes dominate the dynamics $(\alpha>0)$ and (b) when the coupling to nodes with least neighbors is strengthened $(\alpha<0)$. Here, we compute the average standard deviation from a sample of 500 initial configurations and fix $a=2, k=m_{0}=8$ and $N=1000$.
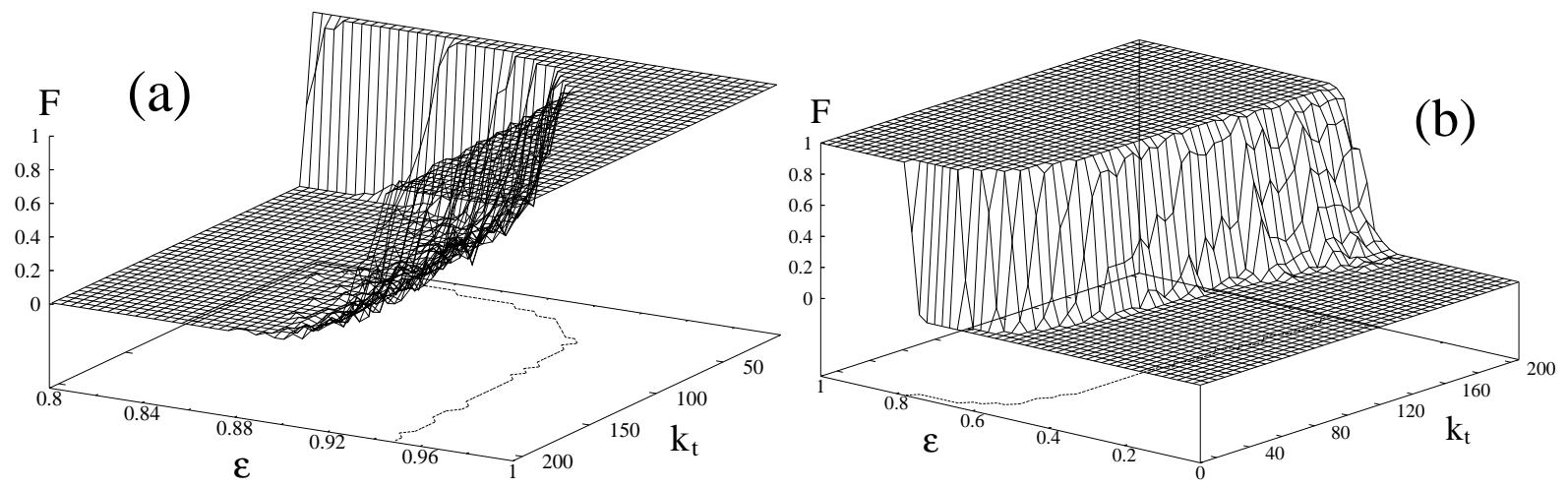

FIG. 6: Transition to coherence when synchronization is imposed to all nodes having a number of neighbors (a) larger than a threshold $k_{t}$, and (b) smaller than $k_{t}$ (see text). Here $a=2, k=8, \alpha=0$ and $N=1000$.

initial configurations and vary the coupling strength and heterogeneity for $a=2$ and $L=1000$. While Fig. 5h shows the numerical results for $\alpha>0$, i.e. in the case where nodes are more strongly coupled to the neighbors with higher connectivities, Fig. [5 shows the transition to coherence when $\alpha<0$. Here synchronizability is observed only for $\alpha \gtrsim-0.15$ and for very high coupling strengths $\varepsilon \gtrsim 0.95$.

We end our study of coherent solutions in random scale-free networks by investigating briefly the role of hubs in the lattice. Instead of strengthening the coupling to the most connected nodes by increasing $\alpha>0$, we now fix $\alpha=0$ and impose synchronization between all the nodes with more than a certain threshold $k_{t}$ of neighbors and observe which fraction of the initial configurations converges to a coherent state. In this case the transition to coherence converges asymptotically to a limit of the coupling strength, as shown in Fig. 61. The same occurs when synchronization is imposed to all nodes with less than $k_{t}$ neighbors, as shown in Fig. $6 \mathrm{p}$.

\section{B. Deterministic scale-free networks}

In the previous Section we focused on random scale-free networks, i.e. growing networks where new nodes are connected following probabilistic rules. In this Section we study deterministic scale-free networks [37, 38, 39], using two different deterministic topologies: the pseudo-fractal scale-free network introduced by Dorogovtsev et al [38] and the Apollonian network introduced by Andrade et al [39] and studied also in Ref. [40]. Both networks are illustrated in Fig. 7]

The pseudo-fractal network of Dorogovtsev is obtained, starting from three interconnected nodes, and at each iteration each edge generates a new node, attached to its two vertices. Figure 7 illustrates this network after three iterations, i.e. with three generations of nodes. The number of nodes $N_{n}$ and the number of connections $V_{n}$ increases with the number of generations as [38]

$$
\begin{aligned}
N_{n} & =\frac{3}{2}\left(3^{n}+1\right), \\
V_{n} & =3^{n+1} .
\end{aligned}
$$

From Fig. 7h one easily sees that this network has indeed a scale-free topology, since the number of nodes 


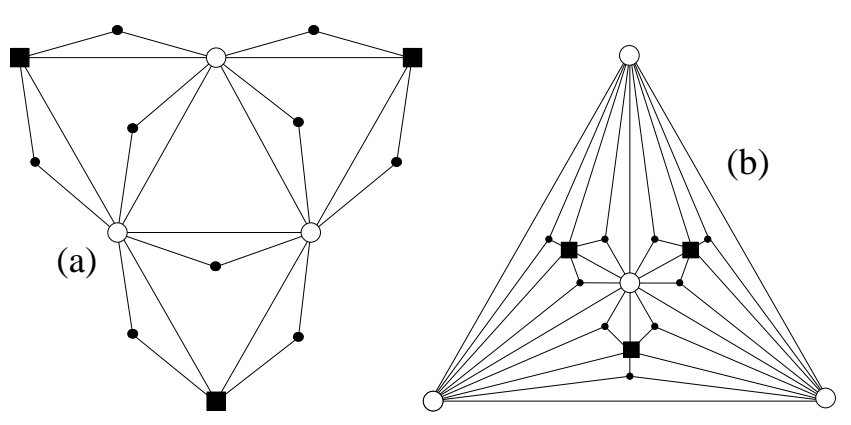

FIG. 7: Illustrations of two deterministic scale-free networks: (a) the pseudo-fractal network [41], and (b) the Apollonian network [39]. Identical symbols label nodes belonging to the same generation $n$ (see text), namely $\bigcirc$ for $n=0, \boldsymbol{\square}$ for $n=1$ and $\bullet$ for $n=2$.

with degree $k=2,2^{2}, \ldots, 2^{n-1}, 2^{n}$ and $2^{n+1}$ is equal to $3^{n}, 3^{n-1}, \ldots, 3^{2}, 3$ and 3 respectively. In particular, the exponent of this power-law distribution is $\gamma=1+\ln 3 / \ln 2$. Moreover, the cluster coefficient of a node with degree $k$ is $C=2 / k$, and the average path length is approximately $\langle\ell\rangle \simeq 4 \ln N_{n} /(9 \ln 3)$.

The Apollonian network is constructed in a different way: one starts with three interconnected nodes, defining a triangle; at $n=0$ one puts a new node at the center of the triangle and joins it to the three other nodes, thus defining three new smaller triangles; at iteration $n=1$ one adds at the center of each of these three triangles a new node, connected to the three vertices of the triangle, defining nine new triangles and so on (see Fig. 7b). The number of nodes and the number of connections are given respectively by

$$
\begin{aligned}
N_{n} & =\frac{1}{2}\left(3^{n+1}+5\right), \\
V_{n} & =\frac{3}{2}\left(3^{n+1}+1\right) .
\end{aligned}
$$

The distribution of connections obeys a power-law, since the number of nodes with degree $k=3,3 \cdot 2,3 \cdot 2^{2}, \ldots, 3 \cdot 2^{n-1}, 3$. $2^{n}$ and $2^{n+1}$ is equal to $3^{n}, 3^{n-1}, 3^{n-2}, \ldots, 3^{2}, 3,1$ and 3 respectively, and the exponent $\gamma$ is the same as for the pseudofractal network. Moreover, a node with $k$ neighbors has a cluster coefficient of $C \simeq 4 / k$ as reported in [40], converging on average to $C_{\infty}=0.828$, and the average path length grows weaker than $\ln N_{n}$ [39].

Despite that both networks have similar values for the topological quantities, they are quite different from the geometrical point of view: the pseudo-fractal network has no metric, while the Apollonian network is embedded in Euclidean space and fills it densely as $n \rightarrow \infty$, being particularly suited for describing geographical situations [39].

For stability analysis purposes (see Section I), the Laplacian matrix $\mathbb{G}$ of deterministic networks can be analytically determined from the adjacency matrix $\mathbb{A}=\left\{a_{i j}\right\}$, since they are related by

$$
\mathbb{G}=\mathbb{I}+\mathbb{A} \mathbb{T}
$$

where $\mathbb{I}$ is the identity matrix and the values of matrix $\mathbb{T}=$
$\left\{T_{i j}\right\}$ are defined by

$$
T_{i j}=-\frac{a_{j i}\left[\sum_{k=1}^{N} a_{i k}\right]^{\alpha}}{\sum_{p=1}^{N} a_{j p}\left[\sum_{k=1}^{N} a_{p k}\right]^{\alpha}} .
$$

A simple way to write the adjacency matrix of the pseudofractal network is

$$
\mathbb{A}_{n}=\left[\begin{array}{cc}
\mathbb{A}_{n-1} & \mathbb{M}_{n-1} \\
\mathbb{M}_{n-1}^{T} & \emptyset
\end{array}\right]_{N_{n} \times N_{n}}
$$

where $N_{n}$ is given by Eq. (7a), $\mathbb{M}^{T}$ represents the transposed matrix of $\mathbb{M}$ and for each generation $n=1,2, \ldots$ the matrix $\mathbb{M}_{n}$ reads

$$
\mathbb{M}_{n}=\left[\begin{array}{ccc}
\mathbb{M}_{n-1} & \mathbb{M}_{n-1} & \emptyset \\
\emptyset & \emptyset & \mathbb{B}_{n-1}
\end{array}\right]_{2 \cdot 3^{n-1} \times 3^{n}}
$$

with

$$
\mathbb{B}_{n-1}=\left[\begin{array}{cccc}
\mathbb{A}_{0} & \emptyset & \ldots & \emptyset \\
\emptyset & \mathbb{A}_{0} & \ldots & \emptyset \\
\vdots & \vdots & \ddots & \vdots \\
\emptyset & \emptyset & \ldots & \mathbb{A}_{0}
\end{array}\right]_{3^{n-1} \times 3^{n-1}}
$$

and whose starting form is

$$
\mathbb{M}_{0}=\mathbb{A}_{0}=\left[\begin{array}{lll}
0 & 1 & 1 \\
1 & 0 & 1 \\
1 & 1 & 0
\end{array}\right]_{3 \times 3}
$$

For the Apollonian network, the adjacency matrix is given by the same recurrence of Eq. 11, but this time with

$$
\mathbb{A}_{0}=\left[\begin{array}{llll}
0 & 1 & 1 & 1 \\
1 & 0 & 1 & 1 \\
1 & 1 & 0 & 1 \\
1 & 1 & 1 & 0
\end{array}\right]
$$

and $\mathbb{M}_{n}$ being a matrix with $\left(3^{n}+5\right) / 2$ rows and $3^{n}$ columns and having in each column only three non-zero elements.

Figure 8 shows the eigenspectra of the Laplacian matrices for both the pseudo-fractal (Fig. 81) and the Apollonian (Fig. 8p) networks, as a function of heterogeneity. As one sees for $a=2$ (solid lines) synchronizability is observed only above $\alpha \gtrsim 1.5$, and in particular there is no synchronizability for the homogeneous coupling regime $(\alpha=0)$. Figure 9 shows the distribution of the average standard deviation over a sample of 500 initial configurations, from which one clearly sees that there are no coherent solutions. Here the standard mean deviation is characterized by some large value which is almost constant beyond the weak coupling regime $(\varepsilon \gtrsim 0.2)$. In the weak coupling regime $(\varepsilon \lesssim 0.2)$ the standard mean square deviation is even larger, since the coupling is not strong enough to compensate the highly chaotic local dynamics $(a=2)$.

From Fig. 8 one also sees that, for the pseudo-fractal and $\alpha>1.5$, the upper threshold $\varepsilon_{U}$ increases monotonically with 


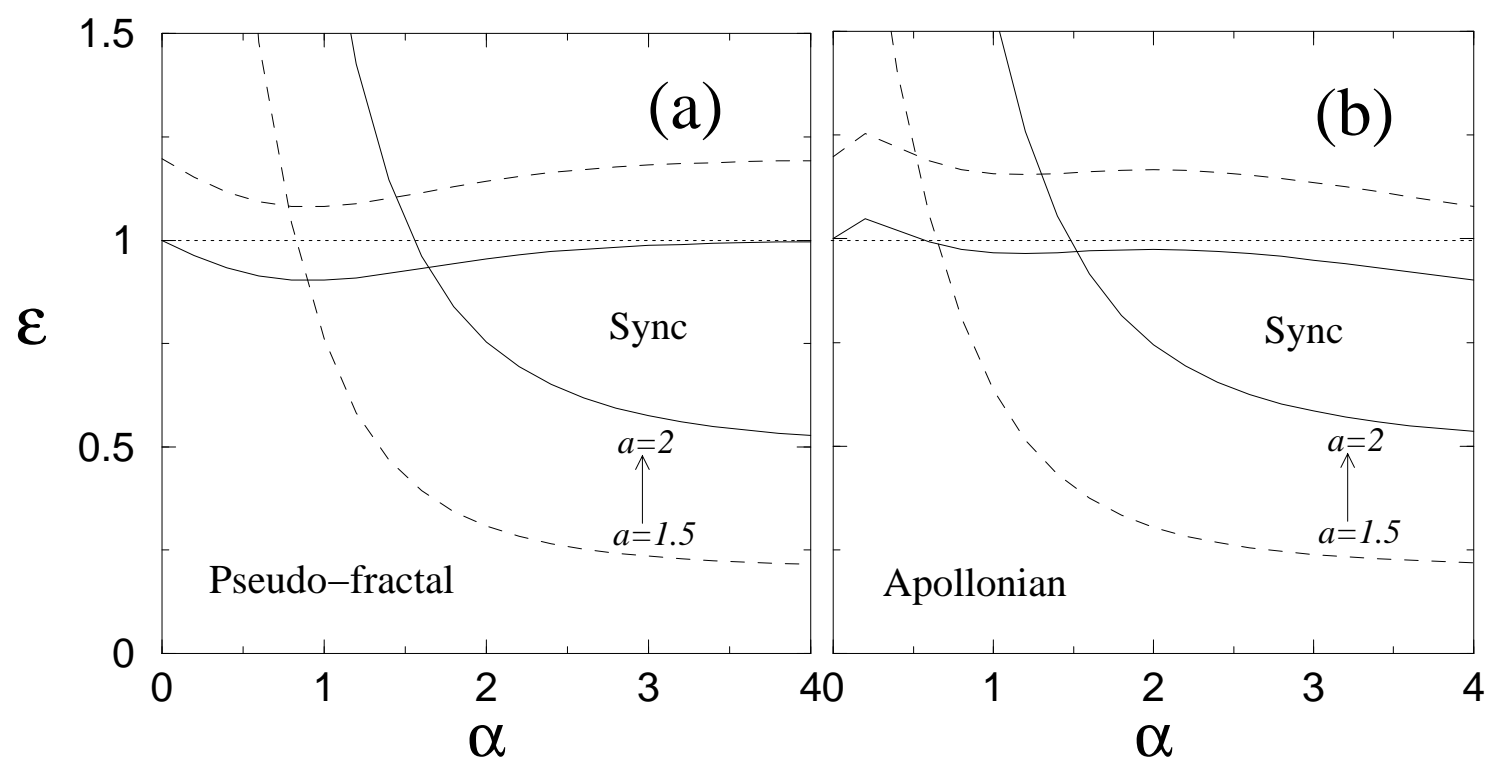

FIG. 8: Boundary $\varepsilon_{L}$ and $\varepsilon_{U}$ for synchronizability as a function of heterogeneity $\alpha$ for (a) the pseudo-fractal network and (b) the Apollonian network, with $a=2$ (solid lines) and $a=1.5$ (dashed lines). For each network we use 6 generations of nodes (see text). These eigenspectra are the same for any number of generations.
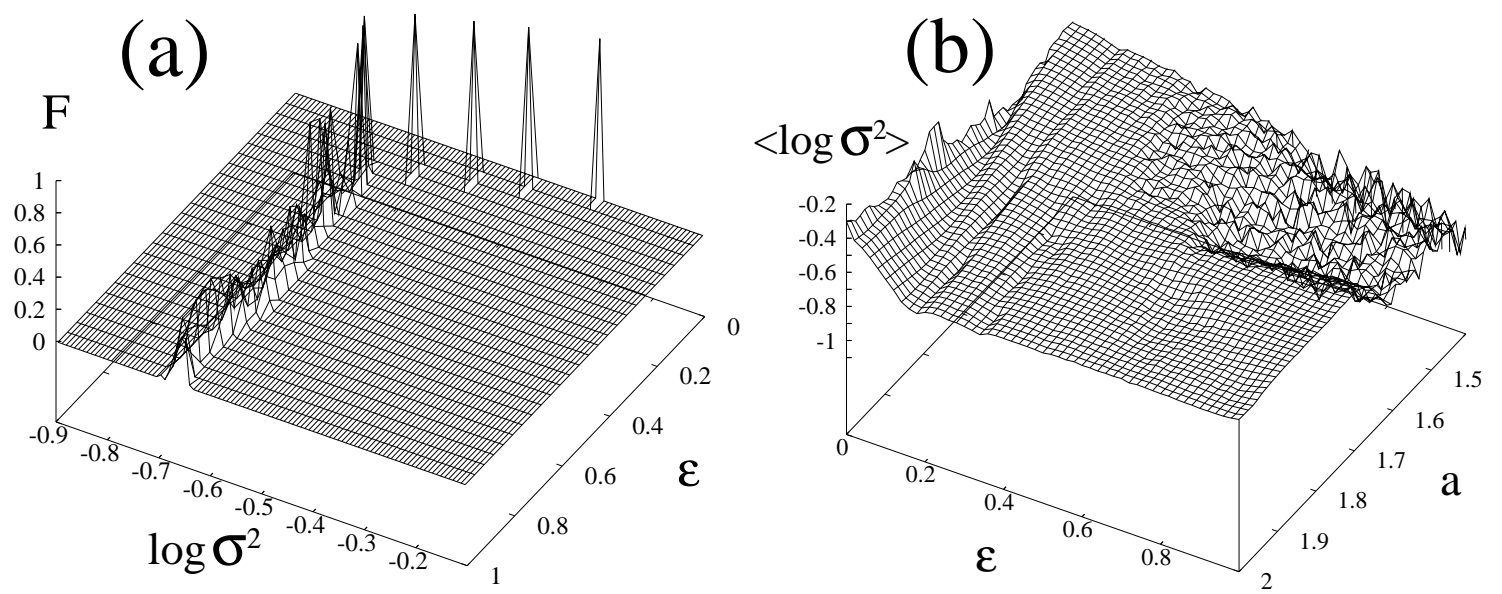

FIG. 9: (a) Typical histogram of the standard mean square deviation $\sigma^{2}$ for the pseudo-fractal network as a function of the coupling strength $\varepsilon$, with $a=2$ and $\alpha=0$. A similar result is obtained for the Apollonian network. (b) Histogram of the standard mean square deviation $\sigma^{2}$ as a function of nonlinearity $a$ and coupling strength $\varepsilon$, for deterministic scale-free networks with $\alpha=0$. The mean square deviation is averaged over a sample of 500 initial configurations and during 100 time steps, after discarding transients of $10^{4}$ time steps.

the heterogeneity, while for the Apollonian network the upper threshold decreases. This particular difference between both networks should be due to their geometrical differences, in particular the fact that Apollonian networks are embedded in Euclidean space could explain in some way that stronger dominance in the coupling to the most connected nodes destroys coherence.

Choosing other values of $a$ for which local dynamics is chaotic, one finds the same values of $\varepsilon_{L}$ and $\varepsilon_{U}$ as functions of $\alpha$ only shifted: $\varepsilon_{L}$ gets smaller, while $\varepsilon_{U}$ increases. Fig- ure 8 illustrates this for the particular case of $a=1.5$. Decreasing even further the nonlinearity below the accumulation point $a=1.411 \ldots$ synchronizability is attained for any positive value of $\alpha$, whenever the coupling strength is sufficiently strong.

Moreover there is a complicated dependence of the average standard deviation on the coupling strength and nonlinearity. As shown in Fig. 90 for deterministic scale-free networks one finds two main regions in the $(a, \varepsilon)$ plane: (I) a region where the standard mean square deviation is large and 

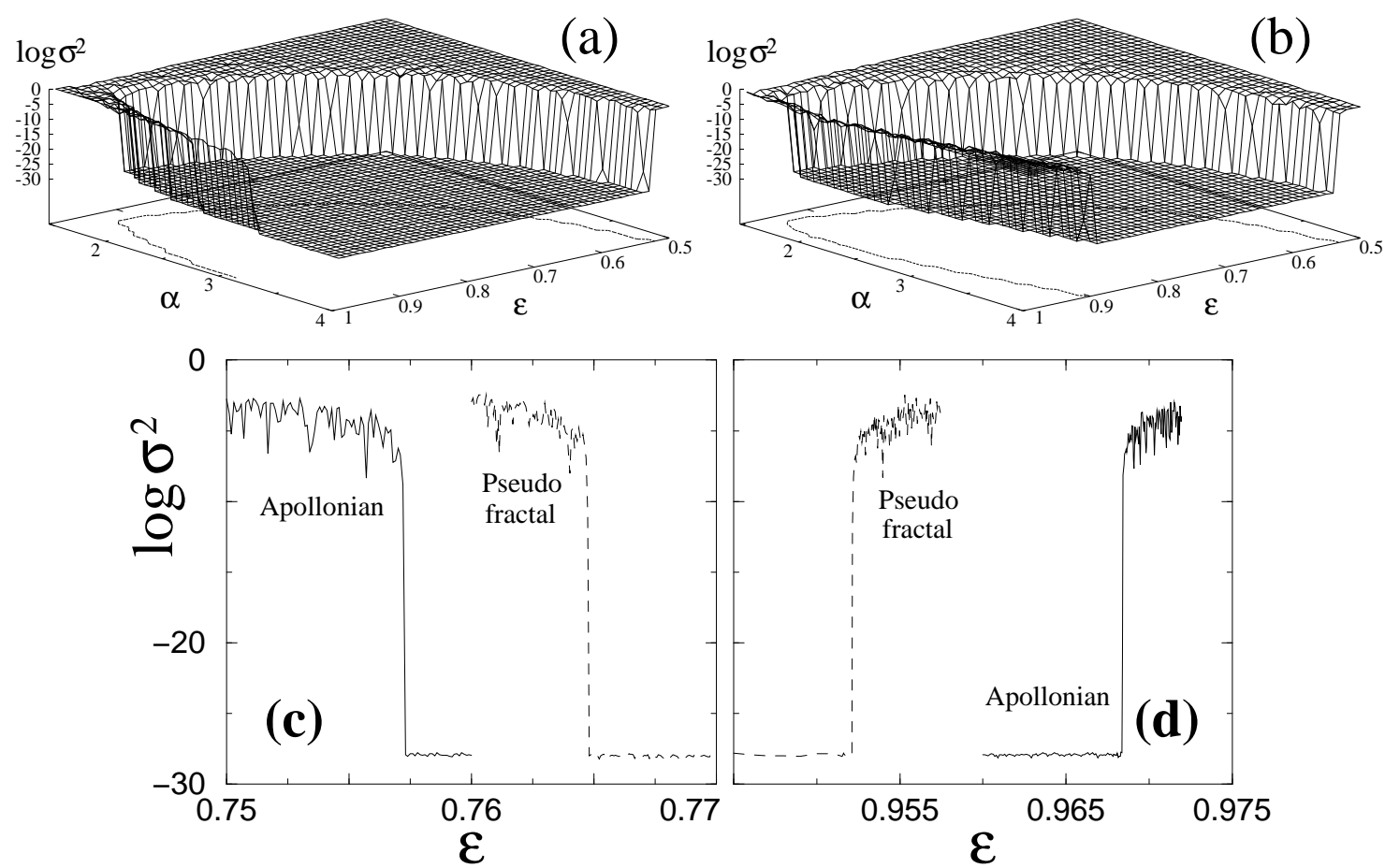

FIG. 10: Inducing transition to coherence by varying the heterogeneity $\alpha$ (see Eq. 11) in scale-free networks. (a) pseudo-fractal network and (b) Apollonian network. For strong heterogeneity coherence appears beyond a relatively high coupling strength, and disappears again for very large couplings (see text). For each network, we use $\ell=6$ generations of nodes and fix $a=2$. (c) and (d) show high-resolution plots of $\sigma^{2}$ as a function of $\varepsilon$ for $\alpha=2$, emphasizing the first-order phase transition to coherence.

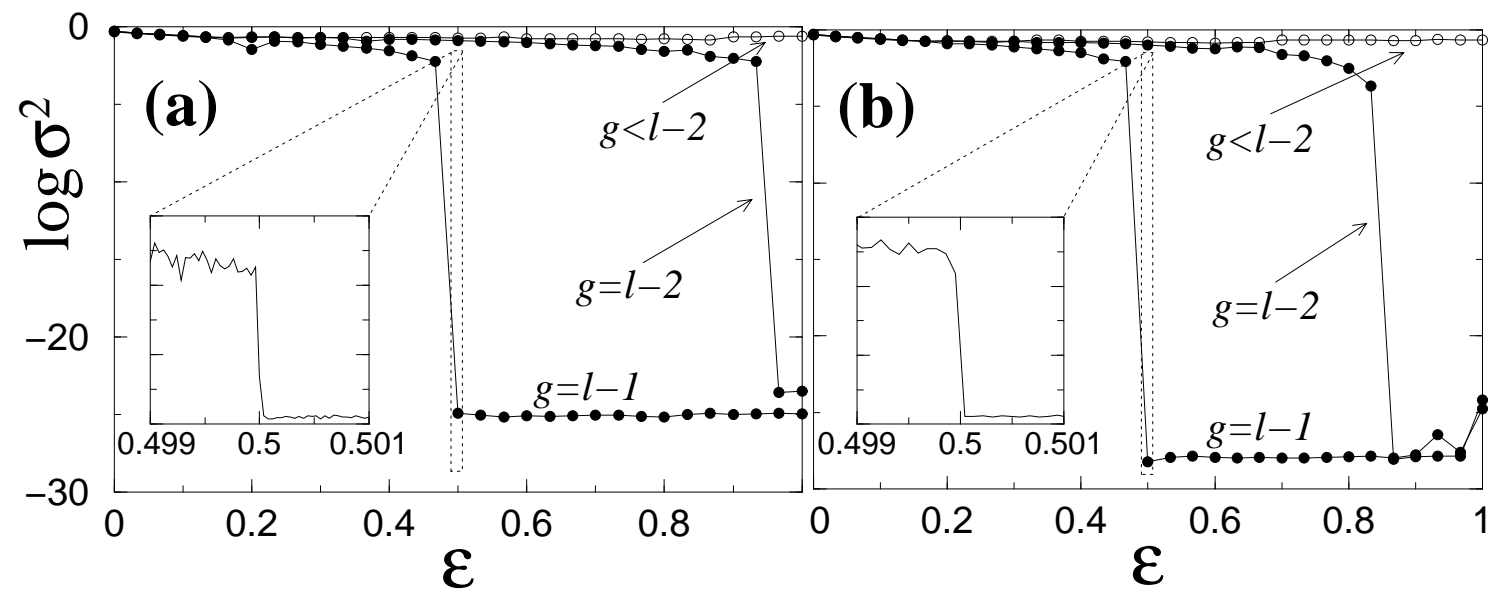

FIG. 11: Transitions to coherence in deterministic scale-free networks, when synchronizing the first $g$ generations of nodes out of $\ell$ generations (see text). (a) pseudo-fractal network and (b) Apollonian network. The collective dynamical behavior is quite insensitive to hubs (see text). Insets show that transitions to coherence are of first-order. For each network, we use $\ell=9$ generations of nodes and $a=2$ fixed.

varies smoothly with the parameters and (II) a region where the mean square deviation is smaller but has larger fluctuations. This second region, observed for $a \lesssim 1.7$, is somehow surprising, since irregular variations of the standard mean square deviation occur for low nonlinearity and high coupling strengths, precisely where one would expect the most regular behavior of the node dynamics.
As for the heterogeneous coupling regime $(\alpha \neq 0)$, Fig. 10 illustrates the transition to coherence by varying the heterogeneity $\alpha$ for the pseudo-fractal (Fig. 10 and the Apollonian network (Fig. 10p). For both networks, one sees that coherence sets in for $\alpha \gtrsim 1.5$, and the contour of the histograms marking the transition to coherence fits well the regions in Fig. 8 labeled as 'sync'. Moreover, from Figs. 10, and 10, 
one observes that all these transitions to coherence are of firstorder.

Finally, we study the role of hubs in deterministic scalefree networks, as we did in the previous Section for random networks. To this end, we impose synchronization among $g=1, \ldots, \ell$ generations, with $\ell$ being the total number of generations, and observed in what conditions coherent states are observed. In the pseudo-fractal network the first generation has $N_{1}-N_{0}=3$ nodes, the second one has $N_{2}-N_{1}=9$ nodes, and the $n$th generation has $N_{n}-N_{n-1}=3^{n}$ nodes. In the Apollonian network the number of nodes appearing at each generation is precisely the same.

Figure 11 shows the standard mean square deviation as a function of coupling strength for pseudo-fractal (Fig. 111) and Apollonian networks (Fig. 11b). In each case we choose the fully chaotic map $(a=2)$ and impose synchronization among the nodes of the first $g$ generations by setting them to be their mean amplitude at each time-step. In both cases, one sees that the standard mean square deviation remains large when synchronization is imposed to all $g<\ell-2$ generations. Coherent solutions are only observed for $g=\ell-2$ and $g=\ell-1$, beyond a coupling threshold which is smaller for the latter case. Surprisingly, for $g=\ell-1$ the transition to coherence occurs for the same coupling strength in both networks. This may be due to the fact that, the fraction $N_{g} / N_{\ell}=3^{g-\ell}=1 / 3$ of nodes on which one imposes synchronization is the same for both networks and is high enough to suppress the influence of local connectivities.

For $g=\ell-2$ the pseudo-fractal network shows coherence only above very high coupling strengths, near $\varepsilon \sim 1$, while for Apollonian networks the threshold is much lower. This difference in the coupling strength threshold is due to the fact that here the fraction of nodes $N_{g} / N_{\ell}=1 / 9$ to which synchronization is imposed is small enough to not suppress the influence of local connectivities. Therefore, since the hubs in the pseudo-fractal network are less connected than the hubs in Apollonian networks, one needs higher coupling strength to observe coherence. For any higher value $\ell$ of generations the same results are obtained, since one has for the quotient of the number of nodes between two successive generations $N_{n} / N_{n-1} \rightarrow 3$ as $n$ increases.

As a general remark, one observes from Fig. 11 that one needs to synchronize a rather high fraction of nodes $(\gtrsim 1 / 9)$ to induce coherence. Therefore, it seems that, dynamical collective behavior on scale-free networks is quite insensitive to hubs. As shown in the insets of Figs. 11] and 11p, the transition to coherence is also of first-order.

\section{DISCUSSION AND CONCLUSIONS}

In this paper we studied fully synchronized solutions for three scale-free network topologies. The main conclusion is the following: in random scale-free networks synchronization of chaotic maps not only depends on the coupling strength but is mainly controlled by the outgoing connectivity $k$, which is a measure of cohesion in the networks. Because of that, one finds coherent solutions in random scale-free networks of fully chaotic logistic maps ( $a=2$ ) with outgoing connectivity $k=8$ and homogeneous coupling, but not in deterministic scale-free networks, since they have rather small effective outgoing connectivity, namely $k=2$ for the pseudo-fractal network and $k=3$ for the Apollonian network. Therefore, although the exponent $\gamma$ of connection distributions in scalefree networks does not depend on the outgoing connectivity [2], we have shown that, in general, synchronization of chaotic maps in such coupling topologies is quite sensitive to it.

Our results were obtained both numerically, from histogram of significantly large samples of initial configurations with a criterion for full synchronization based on the mean standard deviation of amplitudes, Eq. [5], and analytically from the eigenvalue spectra of the diagonalized variational equations computed at the coherent states, Eq. (3).

In particular, for random scale-free networks, the threshold values of the coupling strength obey a power-law, Eq. (6), as function of the outgoing connectivity. The exponent of this power-law depends on the nonlinearity $a$ of the chaotic map, being almost constant below $a_{c} \sim 1.7$ and decreasing linearly above it. Interestingly this value of $a_{c}$ is in the vicinity of the bifurcation of the quadratic map where the period-3 window appears, and coincides with the appearance of other nontrivial behaviors in coupled map lattices with regular topologies, namely in the velocity distribution of traveling wave solutions [24].

For deterministic scale-free networks with homogeneous coupling, the same value $a_{c}$ indicates the threshold above which no coherent solutions are observed, independently of the coupling strength. Above $a_{c}$, coherence is observed only for heterogeneous coupling, namely for $\alpha \gtrsim 1.5$. However, for this range of values, we have also shown that coherence is also absent either for very small or for very large coupling strengths, due to spatial instabilities. Another particularly interesting result that still needs to be explained is that, for Apollonian networks, the coupling threshold beyond which coherence disappears gets smaller when the heterogeneity is further increased. This point is not observed for the pseudo-fractal network and may be due to the geometrical differences between both deterministic networks.

As a general property, we have shown that all transitions to coherence are of first-order, indicating a similarity with other complex networks [29]. Furthermore, all results are robust not only against changes of the initial configurations of node amplitude but also, in random scale-free networks, against changes of the connection network. We also presented results indicating that in scale-free networks hubs play apparently no fundamental role in the dynamical collective behavior.

\section{Acknowledgments}

The authors thank A.O. Sousa and C. Zhou for useful discussions. P.G.L. thanks Fundação para a Ciência e a Tecnologia, Portugal, for financial support. J.A.C.G. thanks Conselho Nacional de Desenvolvimento Científico e Tecnológico, Brazil and Sonderforschungsbereich 404 of DFG for financial support. 
[1] S. Bornholdt, H.G. Schuster (eds.): Handbook of Graphs and Networks (Wiley-VCH, Weinheim 2003).

[2] R. Albert and A.-L. Barabási, Rev. Mod. Phys. 74, 47 (2002).

[3] S.N. Dorogovtsev and J.F.F. Mendes, Adv. Phys. 51, 1079 (2002).

[4] R. Meucci, R. McAllister, and R. Roy, Phys. Rev. E 66, 026216 (2002).

[5] J.H. Snoeijer, T.J.H. Vlugt, M. van Hecke, and W. van Saarloos, Phys. Rev. Lett. 92, 054302 (2004).

[6] M. Otto, J.-P. Bouchaud, P. Claudin, and J.E.S. Socolar, Phys. Rev. E 67, 031302 (2003).

[7] C. Texier and G. Montambaux, Phys. Rev. Lett. 92, 186801 (2004).

[8] H. Tanaka, J. Meunier, and D. Bonn, Phys. Rev. E 69, 031404 (2004).

[9] D.P. Almond and C.R. Bowen, Phys. Rev. Lett. 92, 157601 (2004).

[10] M. Small and C.K. Tse, Phys. Rev. E 66, 066701 (2002).

[11] I. Stewart, Nature 427, 601 (2004).

[12] Y. Moreno, M. Nekovee and A. Vespignani, Phys. Rev. E 69, 055101(R) (2004).

[13] Z. Dezso and A.L. Barabási, Phys. Rev. E 65, 055103 (2002).

[14] M. Compiani, E. Capriotti, and R. Casadio, Phys. Rev. E 69, 051905 (2004).

[15] L. Zhaoping, A. Lewis and S. Scarpetta, Phys. Rev. Lett. 92, 198106 (2004).

[16] P.G. Lind, J. Corte-Real and J.A.C. Gallas, Phys. Rev. E 69, 026209 (2004).

[17] S.H. Strogatz, Physica D 143, 1 (2000).

[18] L.M. Pecora, T.L. Carroll, G.A. Johnson, D.J. Mar and J.F. Heagy, Chaos 7, 520 (1997).

[19] C. Anteneodo, A.M. Batista and R.L. Viana, Phys. Lett. A 326, 227-233 (2004).

[20] M. Cross and P. Hohenberg, Rev. Mod. Phys. 65, 851-1112 (1993).

[21] K. Kaneko and I. Tsuda, Chaos and Beyond (Springer-Verlag, Germany, 2000).

[22] S. Wolfram, A New Kind of Science (Wolfram Media Inc., New York, 2002).

[23] K. Kaneko (ed.), Theory and Applications of Coupled Map Lattices (J. Wiley \& Sons, England, 1993).

[24] P.G. Lind, J. Corte-Real and J.A.C. Gallas, Phys. Rev. E 69, 066206 (2004).

[25] S. Boccaletti, J. Kurths, G. Osipov, D.L. Valladares, and C.S. Zhou, Phys. Rep. 366(1-2), 1-101 (2002).

[26] P. Erdös and A. Rényi, Publ. Math. Debrecen 6, 290 (1959).

[27] B. Bollobás, Random Graphs (Academic, London, 1985).

[28] D.J. Watts and S.H. Strogatz, Nature 393, 440 (1998).

[29] S.H. Strogatz, Nature 410, 268 (2001).

[30] A.-L. Barabási and R. Albert, Science 286, 509-512 (1999).

[31] K. Christensen, R. Donangelo, B. Koiler, and K. Sneppen, Phys. Rev. Lett. 81, 2380-2383 (1998).
[32] M.E.J. Newman and D.J. Watts, Phys. Rev. E 60, 7332-7342 (1999).

[33] A.-L. Barabási, R. Albert and H. Jeong, Physica A 272, 173 (1999).

[34] S.N. Dorgovtsev, J.F.F. Mendes and A.N. Samukhin, Phys. Rev. Lett. 85, 4633 (2000).

[35] L. Kullmann and J. Kertész, Phys. Rev. E 63, 051112 (2001).

[36] P.L. Krapivsky, S. Redner and F. Leyvraz, Phys. Rev. Lett. 85, 4629 (2001).

[37] A.-L. Barabási, E. Ravasz, and T. Vicsek Physica A 299, 559 (2001).

[38] S.N. Dorogovtsev, A.V. Goltsev, and J.F.F. Mendes, Phys. Rev. E 65, 066122 (2002).

[39] J.S. Andrade Jr., H.J. Herrmann, R.F.S. Andrade, and L. da Silva, "Apollonian networks", cond-mat/0406295(2004).

[40] J.P.K. Doye and C.P. Massen, "Self-similar disk packings as model spatial scale-free networks", cond-mat/0407779 (2004).

[41] M.C. González, A.O. Sousa, and H.J. Herrmann, Int. J. Mod. Phys. C 15, 45 (2004).

[42] H. Chaté and P, Manneville, Chaos 2, 307-311 (1992).

[43] P.M. Gade, Phys. Rev. E 54, 64-70 (1996).

[44] S.C. Manrubia and A.S. Mikhailov, Phy. Rev. E 60, 1579 (1999).

[45] J. Jost and M.P. Joy, Phys. Rev. E 65, 016201 (2001).

[46] T. Nishikawa, A.E. Motter, Y.-C. Lai, and F.C. Hoppensteadt, Phys. Rev. Lett. 91, 014101 (2003).

[47] M. Barahona and L.M. Pecora, Phys. Rev. Lett. 89, 054101 (2002).

[48] L.F. Lago-Fernández, R. Huerta, F. Corbacho and J.A. Siguenza, Phys. Rev. Lett. 84, 2758 (2000).

[49] H. Hong, B.J. Kim, M.Y. Choi, and H. Park, Phys. Rev. E 69, 067105 (2004).

[50] F.M. Atay, J. Jost and A. Wende, Phys. Rev. Lett. 92, 144101 (2004).

[51] X.F. Wang and G. Chen, IEEE Trans. Circ. Sys. I 49, 54 (2002).

[52] A.E. Motter, C. Zhou and J. Kurths, cond-mat/0406207 (2004).

[53] P.G. Lind, J.A.C. Gallas and H.J. Herrmann, "Coherence in scale-free networks of chaotic maps", Phys. Rev. E, in print (2004).

[54] L.M. Pecora and T.L. Carroll, Phys. Rev. Lett. 80, 2109 (1998).

[55] K.S. Fink, G. Johnson, T. Carroll, D. Mar and L. Pecora, Phys. Rev. E 61, 5080 (2000).

[56] S.C. Manrubia, A.S. Mikhailov and D.H. Zanette Emergence of Dynamical Order Synchronization Phenomena in Complex Systems, vol. 2 (World Scientific, Singapore, 2004).

[57] E. Ott, Chaos in Dynamical Systems (Cambridge, USA, 1993).

[58] A. Pikovsky, O. Popovych and Yu. Maistrenko, Phys. Rev. Lett. 87, 044102 (2001). 